\title{
Knowledge Artifacts as Bridges between Theory and Practice: The Clinical Pathway Case
}

\author{
Federico Cabitza ${ }^{1}$, Carla Simone ${ }^{1}$, and Marcello Sarini ${ }^{2}$ \\ ${ }^{1}$ Dipartimento di Informatica, Sistemistica e Comunicazione. Università degli Studi di Milano- \\ Bicocca, Italy, \{cabitza, simone\}@disco.unimib.it \\ ${ }^{2}$ Dipartimento di Psicologia. Università degli Studi di Milano-Bicocca, Italy, \\ sarini@disco.unimib.it
}

\begin{abstract}
This paper discusses how Clinical Pathways (CPs) are defined, used and maintained in two hospital settings. A literature review and observational study are combined to illustrate the composite nature of CPs and the different roles they play in different phases of their life-cycle, with respect to the theme of bridging medical knowledge with the related practices by which physicians deal with a specific care problem. We take the case of the $\mathrm{CP}$ as a paradigmatic case to stress the urgent need for an integrated approach with the computer-based support of information and knowledge management in rapidly evolving cooperative work settings.
\end{abstract}

Keywords: Knowledge artifact, Clinical pathway, Electronic patient record, Medical knowledge, Hospital work

\section{Artifacts that put knowledge in practice}

In the last few years the concept of Knowledge Artifact (KA) has been introduced in relation to a whole series of studies on how to support knowledge, as well as its creation, sharing and management. In fact, knowledge is often operationally defined in terms of a meaningful collection of rules, principles, criteria and informative notions that enable people to interpret a given situation, make decisions, solve problems, communicate and cooperate. Rather than focusing on knowledge itself (whatever it is), this notion leads to focusing on what is used when people have to deal with knowledge, i.e. on the physical artifacts that are created to somehow embed and reify the knowledge that is externalized for a particular purpose. Often, the concept of KA is used intuitively as a mere juxtaposition of the concepts of knowledge and artifact. 
This is often done without giving KA a precise connotation with the risk of loosing the understanding of its role in managing knowledge "in action". This is probably due to the fact that in the literature there are several definitions of KA that mainly differ in respect to how the represented knowledge characterizes a specific community of practitioners. Limiting ourselves to the organizational domain, the definition of KA given by Holsapple and Joshi within their Organizational Knowledge Resources Framework [1] emphasizes its generality and ability to be further specialized. In fact, a knowledge artifact is any object that conveys or holds usable representations of knowledge. As any object, KAs can be transferred, shared, and preserved. Moreover, usability of a KA is interpreted as its ability to be put into action by a human actor in an organizational context: however, there is no explanation about how a KA can make this happen. Accordingly, Seiner keeps the characteristic of representability and usability that are intrinsic in the previous definition, but goes one step further. He also stresses the aspect of shareability by defining a KA as any "defined piece of recorded knowledge that exists in a format that can be retrieved to be used by others" [2]. By using the term "recorded" the latter definition hints both to an act of encoding, and also to an accumulation that the knowledge artifact must somehow permit, in order to grow within the community of its users and together with their competencies, experiences and knowledge. The fundamental role of the memory characterizes the acceptation of KA proposed in [3], where the authors focus on how such artifacts support the process of knowledge creation [4] and management due to the fact that they are collectively defined as the result of a progressive stratification of experiences, local practices of use and lessons learned to solve a problem. Then, KAs support practitioners only for their capability of being usable, i.e., to actually be artifacts open to human interpretation and capable of evolving in virtue of the constant negotiation of intended goals, involved incentives and responsibilities within the community.

In light of the last definition, we denote artifacts as knowledge artifacts whenever they are primarily used to objectify how people within an organization and community organize their "memories" and the involved "knowledge" and how people are able to put it into use to make proper and timely decisions. As reported in [3], this can also happen in the presence of underspecified knowledge: in fact, the common ground of the community provides the key to the right interpretation in the given context. This makes underspecification an economical way of maintaining usable knowledge within the community.

Organizational settings, and more generally cooperative settings, provide a wealth of significant examples of the social and participatory nature of the core knowledge involved, as well as of the dynamic and cumulative nature of the knowledge artifacts reifying it: almost any knowledge representation that has been collaboratively edited and that can be updated and annotated as necessary by its "consumers" can be considered a knowledge artifact, as long as it "incorporates" core competencies and "best practice" in which members of a community 
recognize themselves successfully solving problems and adding value to their activities.

In this paper, we focus on Clinical Pathways (CPs) and interpret their role in the hospital setting in terms of KA: in Section 2, we provide a general definition of CPs and briefly discuss their relationship with clinical guidelines. Section 3 discusses the composite nature of CPs and outlines their general structure and the types of knowledge they encompass in terms of different and complementary artifacts. In Section 4, we illustrate the main findings of our observational study with regards to the different ways CPs are used during their life-cycle. Lastly, Section 5 discusses these findings in the light of the advocated integration between CPs and clinical records and, more generally, between knowledge management technologies and actual work practice.

\section{Clinical pathways for the clinical practice}

For its apparent variability, interpretability and context-dependability, medical knowledge is something that cannot be simply learned from university textbooks and by putting practice aside. To this aim, clinicians have always relied on a number of different representations specifically conceived to provide quick and concise access to the relevant procedural knowledge, i.e., knowledge on how to apply notions of human anatomy, physiology and pathology to the pragmatic management of single and peculiar clinical cases. These representations have been called algorithms, protocols, procedures, plans and other similar terms: these terms are endowed with semantic nuances that usually depend on the extent to which clinical context is reported and in what detail, although the related concepts often end up simply overlapping. All of these representations share the idea that an ideal way to cope with a specific health problem exists, whereas ideality relates to the presupposed ability to minimize risks and to optimize chances of full recovery. The medical community achieves and improves on these effective ways of coping with sick patients as part of its daily work. Doctors report on this in specialist publications and literature contributions that are periodically digested and summarized in what they call guidelines, i.e., "a collection of systematically developed statements to assist practitioners [. . . ] for specific circumstances" [5] to cope with their indeterminacy and unpredictability. These statements are expressed in terms of discursive and punctual recommendations towards "best practice" where all non-essential elements from the original context have been expunged to reach the necessary generality.

Clinical Pathways (CPs) have been proposed as a way of combining all the recommendations pertaining to a typical course of illness and of articulating them along the temporal and organizational dimension, i.e., in terms of who does what or when. Notwithstanding the apparent plainness of their function, a recent survey on PubMed literature identified more than 70 slightly different definitions of the 
term "clinical pathway" in more than 500 papers [6]. Most of these definitions define a pathway as an artifact which provides easy and convenient access to the whole body of useful notions that regard a specific treatment plan. The treatment plans are usually agreed upon by a group of clinicians to establish a reference in the management of a particular disease, and hence of any patient who could suffer from this disease, in order to reduce the odds of inappropriate interventions. This ambitious goal is not always reached: actually, what is more often obtained is a reduction of unnecessary variability of treatment that enables a more reliable and precise benchmarking among healthcare facilities. It also fosters the discovery and adoption of clinical evidence due to the better comparability and reproducibility of outcomes.

The reason why a medical staff decides to consolidate "best" or "usual" practices in specific CPs out of their usual interventions generally involves considerations based on either volume, cost, or risk of treatment [7] but also personal interests and academic drives . Usually establishing a CP in a clinical arrangement requires a team of healthcare providers to meet to combine the practitioners' multidisciplinary personal knowledge, usual practices and preferences with the existing medical literature and guidelines. They do that in order to establish what they consider the best treatment for a medical problem in their own settings. In this combination and adaptation to local needs, practitioners tend to consider any aspect that characterizes their own situations, e.g., organizational and resource-related constraints that would make the "ideal theory" of guidelines unfeasible.

\section{Debundling clinical pathways}

In order to have a clear picture of the interrelated aspects that characterize a $\mathrm{CP}$, we undertook an observational study of how clinical pathways are designed, used and maintained in two Neonatal Intensive Care Units (NICU) of two important hospitals in Northern Italy, respectively the 'Alessandro Manzoni Hospital' in Lecco and the 'Giovanni Fornaroli Hospital' in Magenta (in the following denoted as NICULe and NICUMa, respectively).

We decided to focus on two NICU settings for a number of reasons. Neonatology is still a young discipline devoted to life-saving care of ill and premature newborn infants. Like in other intensive care disciplines, the practitioners involved in neonatology are faced with challenging standards with respect to efficiency, timeliness and effectiveness of intervention. Even more than in other similar disciplines, the increasing effectiveness of neonatal interventions relies on constant and continuous innovation, in regards to both technological equipment (e.g., mechanical ventilation), drug treatment (e.g., pulmonary surfactant replacement) and process improvement. This orientation towards continuous improvement and modernization has dramatically improved the 
survival rates of extremely premature infants and decreased the rates of disabling complications. For our studies, we undertook ethnomethodological observations in a number of hospital settings, including geriatrics, cardiology, orthopedics and internal medicine departments. Yet, neonatologists were the practitioners who showed the most enthusiasm towards computer-based support for their work and who were willing to consider their own practice from an objective point of view. We decided to focus on their work setting and practices, since we observed how neonatologists were deeply and sincerely committed to taking full advantage of the use of clinical pathways as a concrete and time-saving artifact that could actually improve their practice.

Indeed, both in the NICULe and NICUMa we saw that CPs were a significant case of composite knowledge artifact. In fact, the CPs we studied contained a wealth of documents, sheets, sections, maps, diagrams and forms [8, 9]. These heterogeneous artifacts differ for a number of reasons: e.g., because they bear different amounts of description and specification or they have been conceived for different purposes by practitioners with different competencies. They have been put together in order to provide practitioners with all the necessary notions and tools to make the "care plan" clear, to recognize its applicability to a specific clinical case and to help them in keeping the care trajectory "on track" with respect to that plan. Usually a CP is a collection of pre-existing documents of this kind that are put together to support a process of care "as a whole" by providing convenient access to a heterogeneous palette of indications related to the procedural, organizational, and medical knowledge that is available in a specific hospital setting, aimed at directly supporting different phases and episodes within the intended path. A significant example of these enclosures are the nomograms: graphical tools that support the predictive ability of practitioners which are intended to reify a sort of statistic knowledge about odd ratios and diagnostic / prognostic probability of test outcomes. Besides the nomograms, a CP can also encompass templates and structured forms: they provide an obvious support for compilation and data collection, but they also reify the knowledge indicating the minimum set of data to consider in order to undertake an intervention that is compliant to the law and local norms. Likewise, numerical scales and thresholdbased criteria reported in the CP can be seen as cognitive aids conveying knowledge about the optimal heuristics to make clinical decisions that reduce the risk of adverse events. Classification schema and taxonomies succinctly reported at the end of a $\mathrm{CP}$ are intended to provide practitioners with indications regarding what conceptual categories must be considered in the interpretation of relevant clinical phenomena. To this same aim, even simple check-lists can be seen as tools conveying knowledge about what steps are to perform and what facts to consider in what loose order as a general and not prescriptive rule. Since activities and supportive tools are chosen according to the latest and most reliable guidelines, CPs are also endowed with references or short excerpts of those single recommendations from the guidelines that are applicable in each step of the 
pathway. Most of the time, recommendations are reported along with the strength of the related clinical evidence and degree of reliability.

Besides these knowledge-oriented components, CPs also encompass sheets which are purposely conceived to help practitioners to either monitor the performance of the care process with respect to specific output indicators extrapolated from the guidelines, or to keep track of the occurrence of variances during the progress of the illness of the patient with respect to the intended plan. Despite other names used in the literature, we refer to these artifacts as either monitoring forms and variance records, respectively. Variance records are those specific artifacts where clinicians are supposed to report "all the unexpected events which occur during patient care events which are different from those predicted in the pathway" [10]. Monitoring forms are artifacts by which clinicians can collect data needed to monitor the output of the most critical activities that are associated to performance indicators (e.g., number of times the patient has received a specific treatment in the last 48 hours with respect to the standard indicated by the guidelines). Both variance reporting and data monitoring are activities intended to facilitate the aggregation of data from multiple applications of the same pathway and enable its post-hoc analysis. This latter activity allows for the progressive tuning of the $\mathrm{CP}$ with respect to either the latest clinical evidences or the local best practices as we will see in more detail in the next section.

\section{Clinical pathways in action}

Once the composite structure and intended goals of a CP were identified, the next step of the study was to understand the actual role of CPs within medical practice in the two hospital wards we studied. The interviews, observations and their analysis highlighted four different phases where CPs play a different role: creation, use, evaluation, and update.

\subsection{CP creation}

The physicians and nurses interviewed at the NICUMa told us that the decision to embark the process of definition and adoption of a new CP is usually driven by two alternative criteria: frequency of cases and seriousness of illness. For example, gastroenteritis was chosen since it is a frequent disease in newborns and infants, although the problems that it causes are not severe. However, the high number of cases requires some shared and agreed criteria that can be expressed within a CP in order to admit patients only when they really require hospitalization. On the other hand, the criterion of seriousness was, e.g., applied 
when there was an unexpected outburst of meningitis cases at the hospital. In such a critical situation, decisions must be made quickly, often in an emergency context: therefore, the clinicians decided to make a trustworthy reference available to all staff involved and hence put it into a corresponding CP for meningitis.

The definition of a CP can be framed into the more general issue of resistance to changes. The problem of resistance to changes in healthcare settings is well acknowledged in literature (e.g., [11]). To deal with this issue, some strategies are recommended: in particular i) to not involve all practitioners in innovation, but only the so called "early adopters", i.e. only practitioners who have a positive attitude towards it; ii) to make early adopters' activity visible so that more prudent practitioners can also realize the effects of innovation. This last point is relevant in order to reduce the resistance to change of the more skeptical adopters. We observed similar strategies also in the hospitals under study. Our observations confirmed that the definition of a $\mathrm{CP}$ is a social process involving some representatives of the practitioners working in a specific hospital unit where the need for a CP is perceived. Then CPs are tested by these representatives and successively explained to the rest of the staff. During the definition process, the possibly discordant local practices that clinicians rely on when dealing with a well defined problem are discussed and confronted in the light of the current scientific evidences regarding the particular pathology. This process is not free of problems even if it is carried out by practitioners who have worked together for a long period. We observed three paradigmatic possible outcomes: i) practitioners reach an agreement about a local practice, which possibly conforms to the theory expressed by the guidelines; ii) an agreement cannot be easily reached: in this case, the indications given by the related theory are considered as a sign of a controversial point to be further discussed; iii) the compromise was the outcome of an asymmetric relationship that gave more influence to one of the discussants involved. In the following, we outline three vignettes illustrating the above listed different levels of agreement. The first two cases are about the CP designed for the treatment of neonatal infections due to the beta hemolytic streptococcus group B (GBS), which is often associated in severe gastroenteritis; the last case involves the definition of a $\mathrm{CP}$ to manage cardiac decompensation.

The first vignette illustrates how the theory (guidelines) was "arranged" by clinicians on the basis of a set of locally agreed best practices that are usually deployed to deal with the GBS problem. The head physician of the NICULe told us that the definition of the CP required the identification of what tests should be prescribed in order to formulate a sound diagnosis of the GBS infection. While the related international guidelines suggest the prescription of a wide battery of tests, physicians agreed on the usual practice to prescribe the complete battery of tests only for non-routinary cases, when further investigation is needed. In fact, since some of the recommended tests are too invasive for the infants, physicians preferred to save them from pain and possible complications unless strictly necessary. The structure of the $\mathrm{CP}$ reflected this choice: the first activity prescribes the first subset of tests, then a medical assessment has to be performed; 
if a clear diagnosis can not be formulated, another activity recommends the other (more invasive) tests. The choice to prescribe the complete battery of tests only for non-routinary cases also satisfied the requirement of cost reduction advocated by the hospital administration, since the most invasive tests are often also the most expensive ones. Notwithstanding, this criterion was not considered to be the leading one, but simply a positive addendum.

The second vignette illustrates a case in which participants were not able to reach an agreement. The NICULe head physician described the case in which the physicians involved had discording opinions (even if with slight differences) about which was the best time interval to keep the newborn under observation in order to understand whether the signs of GBS infection had manifested or not. This time is crucial in order to avoid unnecessary treatments that can negatively impact the infants' health. Since after a lengthy discussion an agreement was not reached, the 48-hour time span proposed by the guidelines with strong evidence helped them to overcome this deadlock and deliver the CP that was to be timely issued. Physicians decided to conventionally use that time span as a purely hypothetical value indicating a still open issue to be further investigated. This conventional use has been possible because it was fully under the control of a coherent community, whose members work at arms-length, and continuously confront the practices used to deal with the different clinical situations. In any case, the selected value also protected the patients from too severe drawbacks, should the CP be used by occasional practitioners or novices, and it also protected clinicians from legal liabilities.

The last vignette illustrates a case where the $\mathrm{CP}$ was defined but turned to be practically unusable. The case regards the definition of a CP to manage cardiac decompensation, a condition that necessarily involves different wards and specialties. In this specific case, clinicians belonging to two groups (cardiologists and internists) conducted the negotiation in a way that raised an irreconcilable conflict: on the one hand, the cardiologists wanted to consider the ECG as a routinary exam to formulate a diagnosis for cardiac decompensation and wanted to build the $\mathrm{CP}$ accordingly. On the other hand, internists did not deem this exam as necessary, both for clinical motivations (i.e., other simpler, almost equivalent, diagnostic exams are available) and for organizational reasons: in their ward the ECG machine is not available, and hence performing this exam would require time-consuming and expensive interactions with external facilities. This conflicting situation led to discussions that frustrated the physicians of the internal medicine ward: they quit their active participation in the definition of the CP, whose final version was written taking into account only the cardiologists' point of view but basically never came into practice. This case shows how crucial and fragile the identification of who has to be involved in the definition (and subsequent use) of a CP is. The presence of a common ground is a fundamental precondition that cannot be surrogated by the simple fact that a group of people is dealing with the same disease on the same group of patients. One could object that this happened only because the $\mathrm{CP}$ concerned an inter-ward process. This is not 
completely true: the practitioners interviewed reported similar failures that also happened when a single ward was involved, typically when the head physician did not pay enough attention and effort to establish the right preconditions for the $\mathrm{CP}$ definition, or when a CP defined for a ward was transported as is into an apparently similar one. Therefore, when looking at a $\mathrm{CP}$ as a knowledge artifact, locality either in terms of background and elaboration, plays an unavoidable role.

\subsection{CP in use}

When observing and interviewing practitioners about CPs put in action, the basic question is: do CPs outlive their definition or do they disappear as soon as they have played the role of supportive artifact of the externalization of tacit knowledge? And, in the positive case, are they used only by newcomers or also by the people who built them? In our investigations we had a clear indication that CPs are not "dead letter". In fact, the physicians interviewed reported to us that they take CPs into constant consideration during their work: CPs are usually printed and kept by physicians as separate and unofficial artifacts that complement those pertaining to the clinical record. This habit shows a continuous need of practitioners to be supported in bridging the gap between theory and practice, to have a memory ready-at-hand of how to perform usual or critical practices when for any practical reason the content of this memory is difficult to be aptly retrieved and timely applied to the current case.

CPs do not play a role only for the individual decision-maker. Our observations highlighted how the participatory way in which CPs are defined (when successful), as well as their pervasive presence in the ward, facilitates the creation of a more collaborative relationship between doctors and nurses. From this point of view, CPs can be seen as boundary objects between these two complementary roles [12]. From doctors to nurses, they convey medical knowledge that helps the latter ones to better understand and contextualize doctors' decisions and their follow-up in the activities that nurses perform. The other way round CPs support the transfer of information pertaining to the decision of a doctor to the colleagues who will assess the same patient later. In this specific case, CPs define a clinical context to which this information can be related and become more reliable from the doctor's point of view. As the head-nurse of the NICULe told us: "by means of CPs, nurses are empowered to act as active and prompt reminders about activities due while surveying cases during the ward round and they become more confident in their ability to assist doctors on specific activities".

CPs are not used only as a part of and in combination with the whole clinical record: at the NICULe, a small number of main reference CPs are also printed and pinned on the walls of the small kitchen that physicians use for their informal meals and meetings. Sometimes discussions spring up in front of the CP diagram during coffee breaks, especially in regards to the most interesting cases which 
occurred a few hours earlier [13]. The presence of CPs hung on the walls of the dinette seem to facilitate storytelling and the exchange of experiences, as well as foster the exchange of opinion among peers who have dealt with similar cases: to this aim, CPs constitute a map where critical decisions are located and their consequences are analyzed in a discursive and visual manner.

The usefulness of CPs to deal with concrete problems (either routine or not) is acknowledged by most of the practitioners: notwithstanding, the head physician of NICULe clearly stated to us that CPs are still used as an "external" reference and hence their inclusion in the current practices is not facilitated at present: this is still perceived as an open issue even for those CPs that are actively used. The most advocated solution is to make CPs an official artifact that is fully and seamlessly integrated within the clinical record so that it is possible to more easily draw correlations between the entries of the clinical record and the activities referred to by the CP, and viceversa. This recurrent requirement opens up new possibilities to conceive feasible ways to introduce a computer-based technology supporting the full usage of clinical records: we will come back to this point in the concluding section.

\subsection{CP evaluation and update}

The deeply contextualized nature of CPs makes them a live artifact that evolves together with its context. This evolution can be defined in terms of both evaluation and update: these two phases are strongly correlated since the evaluation gives indications for the changes characterizing the latter and the updating phase justifies the additional effort involved in the former for the sake of continuous quality improvement. In this twofold phase, CPs play two complementary roles with respect to their capability of bridging theory and practice. On the one hand, CPs allow new medical knowledge to come into practice. In fact, once the CP has been consolidated, its updating is triggered by the appearance of new scientific evidence in the specialist literature for the pathology at hand. In this case, the responsible for the $\mathrm{CP}$, a role that is usually defined among the physicians who are expert in that pathology, has to update the CP and notify the updates to the other colleagues accordingly. This usually happens as soon as new evidence become available which contrast or only complement the indications reported in the current CP. Even when new evidence is not available, a revision on average every two years is a common practice in the settings we have studied. On the other hand, CPs facilitate the monitoring of how current behaviors within the ward comply with the intended local best practices embedded in the CPs; CPs also permit the post-hoc evaluation of the performance of the overall caring process that they describe. These two kinds of information should be collected in specialized components of the $\mathrm{CP}$ : the variance records and the monitoring forms we mentioned in Section 3. While the first role traces back to the themes concerning 
the CP definition, the second role raises more serious problems when CPs are put into action. In fact, the filling in of the two above mentioned components is a typical case of effort that in theory is well understood and accepted by all of the involved practitioners, but which in practice is difficult to perform because of the chronic lack of resources shown by the medical settings in Italy. To this regard, the head physicians see the introduction of technological solutions for the management of care information as a way of facilitating physicians in compiling the necessary forms at the place and time of the execution of the activities. This returns to the point that CPs and clinical records need to be more integrated.

At NICUMa, the problem to have information about monitoring behaviors and evaluating performances collected on different specialized components of CPs led to an original solution. Variance records and monitoring forms have been unified into a single form in order to concentrate the compilation effort and to keep some of the context of the inscriptions. The driving goal was to monitor the performance of the process in terms of outputs and of their compliance with the standard indications contained in the CPs. For this some performance indicators have been identified within the organizational unit where CPs are used and inserted in the monitoring form: those indicators refer either to clinical aspects of the care, e.g. whether an antibiotic has been administered in the presence of bacteria for meningitis within the first hour since admission; or to more organizational aspects: e.g., whether a patient has been observed for a few hours after having slight dehydration in gastroenteritis cases; or to more patient specific topics: e.g., whether relatives received clear recommendations about the proper dietetic regimen to follow after their child has been discharged from the hospital. Each indicator has associated a reference value as specified by the local interpretation of the standard values posed by the theory (i.e., the guidelines). The same form contains sections where the free-text description and justification of the deviation from those values have to be inserted. This local solution has several advantages. First, the indicators point to specific activities or their pre/postconditions that are deemed as particularly critical in the process map contained in the related form of the composite CP: this information supplements the process description since it gives a measure of criticality of the activities it contains [9], although the correlation is only implicit. In fact, the two kinds of information are contained in different forms and the connection is dynamically reconstructed only in their practical use. Second, practitioners are better supported in the documentation of the variances. In fact, the presence of all the information on the same sheet lets the above criticality indicate which variances are really relevant to be traced and makes the documentation of the possible variances contextual to the activities and indicators to which they refer to, besides the obvious advantage of avoiding unproductive repetition of the same information on two sheets [14]. When the indicators show that current practice for a particular disease is not performing as well as indicated in the related standard and that the variances occurred exceed a physiological amount (due also to the initial tuning of a new CP), the responsible of the CP goes through the justifications reported therein and decides whether 
measures have to be taken to improve compliance with the practices indicated in the $\mathrm{CP}$, or the latter one has to be modified according to the new current behaviors. The head physician of the NICUMa, who is responsible for the CPs, told us that the strategy is to gather all practitioners together once a month and to call a meeting where practitioners are informed about the indicators whose values do not reach the standard values. These meetings are also intended to facilitate the promotion of knowledge internalization and externalization; in fact, disseminating knowledge about those indicators that fall short of the standard is a way to promote self reflection (internalization) and to raise discussions among the practitioners involved (externalization) in order to find an agreed way to improve team performance with respect to the problematic activities. In all of the considered settings, evaluation and tuning is perceived as a collaborative process that is worth taking up part of a clinician's scarce time for the positive impact on the cohesion of the clinical community and for the improvement of the caring process with respect to patients and their families.

\section{Implications on technologies}

The paper outlined the main findings of a study concerning the definition, usage, evaluation and updating of CPs in two hospital wards. The study showed that this kind of composite knowledge artifact is a carrier of that procedural knowledge that combines the indications of an ideal behavior, as defined by guidelines derived from clinical research, with the constraints imposed by the current setting adopting the CP. Moreover, our study outlined some positive and problematic issues that arise when these knowledge artifacts are put into action. These positive and negative aspects concern the more general issue of how to reconcile the ways practitioners manage data in their daily work with the ways practitioners manage the knowledge they need in order to make sense of the data. This relationship is often problematic, especially since it is generally neglected in the design and development of computer-based information systems (ISs) and knowledge management systems (KMSs). In almost all the application domains, work is based on a set of core pieces of knowledge and on data that are used and produced in virtue of (and according to) that knowledge. Yet, knowledge and data are seldom considered tightly bound together as they are in practice, either by the management of an organization or by the designers of the organization's information system. Within the same organization, the technological solutions dealing with both aspects, ISs and KMSs respectively, are usually under the responsibility of different departments, or, in any case, within different programmes of work automation. This usually results in interoperable components which are logically disjointed. Our point is that if ISs and KMSs look at data and knowledge from irreconcilable perspectives, they end up by forcing behaviors that are hardly mutually supportive of cooperative and knowledge work. 
We are well aware that it is dangerous to propose generalized approaches intended to fit every context, especially when knowledge management is involved. Notwithstanding, the healthcare domain is a paradigmatic case where the production of data and the access to the related knowledge are naturally combined in everyday practice. In fact, data and knowledge are managed by the same community of heterogeneous practitioners who closely interact with each other around the same set of patients and the same set of caring problems. The point we make here is that the development of both ISs and KMs has to take into account this obvious, but usually neglected, fact: actors perceive and need these systems to be integrated as two sides of the same coin. They are not that integrated, especially in all those cases in which data disappear quite quickly after their production either in the rows of a database or in the verbose pages of an almost unusable documentation. They are also not integrated in any other case where the constant and progressive stratification of experiences, solutions and best practices is not fostered or, worse yet, is practically hampered. In these cases, designing towards a strongly interconnected system is almost impossible and the use of looselycoupled and partial solutions seems unavoidable, at least at the initial stage of introduction of KMSs. In other cases, the integration between data and knowledge occurs already in the everyday field of work, where practitioners are also supposed to use consolidated information management technologies that have a strong cultural and pragmatic impact on how they have their work done: here, the integration of knowledge management functionalities could be proposed on top of these existing systems and more importantly, could evolve jointly with them. This is an opportunity we detected for the healthcare domain [15], where Electronic Patient Records (EPRs) were introduced years ago and are being increasingly used in the hospital domain. Since the introduction of a new EPR in a hospital setting is still problematic [16], and since the relevance of CPs as a means to improve the quality of care is constantly increasing among the practitioners of that domain, this is a unique occasion to face the two sides of the same coin within a unified project. In such a project, the interaction functionalities that are typical of both ISs and KMSs could be jointly designed. In this light, EPR would stop looking as a set of templates reflecting the tables of the underlying database and it would become a more flexible, context-aware, and user-centered support that would also encompass knowledge-oriented tools as CPs are. These, in turn, would become closely integrated with clinical data, easy to be updated and tuned, and endowed with a dynamic interface integrated with the EPR that could support timely decision making, and make quality assessment and monitoring a reasonable effort. The above considerations have inspired both our methodological [17] and designoriented [15] researches so far towards the fulfillment of the urgent requirement of integration which we collected from our participatory and observational studies in the last three years in the hospital domain.

Acknowledgements: The work presented in this paper has been partially supported by the Italian fund F.A.R. 2007. The authors would like to thank the 
management and personnel of the Neonatal Intensive Care Units of the Alessandro Manzoni hospital of Lecco and of the Giovanni Fornaroli hospital of Magenta for their kind collaboration.

\section{References}

1. C.W. Holsapple, K.D. Joshi, Organizational knowledge resources, Decis. Support Syst. 31(1), 39 (2001)

2. R. Seiner, Metadata as a knowledge management enabler, TDAN.com \& KIK Consulting Services The Data Administration Newsletter. 15 (2001)

3. S. Bandini, E. Colombo, G. Colombo, F. Sartori, C. Simone, The role of knowledge artifacts in innovation management: the case of a chemical compound designer, Communities and technologies, pp. 327-345 (2003)

4. I. Nonaka, H. Takeuchi, The Knowledge Creating Company. Oxford University Press, Oxford, UK (1995)

5. M. Field, K. Lohr (eds.), Guidelines for Clinical Practice: from development to use. Institute of Medicine, National Academy Press, Washington, DC (1992)

6. D. Bleser, Depreitere, D. Waele, Vanhaecht, Vlayen, Sermeus, Defining pathways, Journal Of Nursing Management 14, 553 (2006)

7. T. May, Clinical pathways: Cure guide or cost-cutter?, Business First of Columbus (1997)

8. F. Cabitza, M. Sarini, On the pathway towards ict-support for a better and sustainable healthcare, In ECEH07 Proceedings of the second European Conference on eHealth, Lecture Notes in Informatics -GI-Edition, vol. P. Gesellschaft fur Informatik, pp. 89-100 (2007)

9. F. Cabitza, M. Sarini, C. Simone, Providing awareness through situated process maps: the hospital care case, In GROUP'07: Proceedings of the 2007 International ACM SIGGROUP Conference on Supporting Group Work, ACM Press, pp. 41-50 (2007)

10. J. Cheah, Clinical pathways - an evaluation of its impact on the quality of care in acute care general hospital in Singapore, Singapore Medical Journal 41, 335 (2000)

11. D.M. Berwick, Disseminating innovations in health care, JAMA 289(15) (2003)

12. S.L. Star, G. Bowker, Sorting Things Out: Classification and its Consequences (MIT Press (1999)

13.D.G. Bobrow, J. Whalen, Community knowledge sharing in practice: the Eureka story, Reflections, the Journal of the Society for Organizational Learning 4(2), 47 (2002)

14.F.. Cabitza, M. Sarini, C. Simone, M. Telaro, "When once is not enough": The role of redundancy in a hospital ward setting, In GROUP'05: Proceedings of the International Conference, ACM Press, pp. 158-167 (2005)

15. F. Cabitza, C. Simone, “... and do it the usual way”: fostering awareness of work conventions in document-mediated collaboration, In ECSCW'07: Proceedings of the Tenth European Conference on Computer Supported Cooperative Work (ECSCW), pp. 119-138 (2007)

16. M. Berg, Health Information Management, Routledge (2003)

17.F. Cabitza, C. Simone, A language for executable specification of coordinative functionalities for electronic document systems, In CHItaly'07, Proceedings of the Fifth Symposium on Human-Computer Interaction (2007) 